\title{
Is It Radiculopathy or Referred Pain? Buttock Pain in Spinal Stenosis Patients
}

\author{
Dae Moo Shim, MD, Tae Gyun Kim, MD, Jun Sung Koo, MD*, \\ Young Ho Kwon, MD*, Chang Su Kim, MD* \\ Department of Orthopaedic Surgery, Wonkwang University Hospital, Iksan, \\ ${ }^{*}$ Department of Orthopaedic Surgery, Kosin University Gospel Hospital, Busan, Korea
}

Background: Buttock pain is common, and there are no fixed guidelines for its diagnosis and treatment. This study compared a selective nerve root block and a facet joint block for patients with degenerative spinal disease and buttock pain.

Methods: Patients with degenerative spinal disease who presented with buttock pain, received a selective nerve root block (group A) or a facet joint block (group B) from June 2017 to September 2017, and were able to be followed up for more than 3 months were prospectively enrolled. Clinical results were assessed using a visual analog scale for comparative analysis.

Results: One day after the procedure, an excellent response was found in $7 \%$ and $6 \%$ of groups $A$ and $B$, respectively; a good response was found in $41 \%$ and $13 \%$ of groups $A$ and $B$, respectively. Two weeks later, an excellent response was found in $11 \%$ and $4 \%$ of groups $A$ and B, respectively; a good response was found in $41 \%$ and $20 \%$ of groups A and B, respectively. Six weeks later, an excellent response was found in $11 \%$ and $7 \%$ of groups $A$ and $B$, respectively, and a good response was found in $41 \%$ and $20 \%$ of groups $A$ and $B$, respectively. At the final follow-up, more than $47 \%$ and $46 \%$ of patients showed a good response in groups $A$ and $B$, respectively. In group $A$, the visual analog scale score improved compared to the pre-procedure value of 5.01 to 2.74 on day 1, 2.51 at week 2, 2.38 at week 6 , and 2.39 at week 12. In group B, the visual analog scale score improved compared to the preprocedure value of 5.24 to 3.94 on day 1, 3.99 at week 2, 3.24 at week 6 , and 2.59 at week 12 . On day 1 and at weeks 2 and 6 , group A showed a significantly better outcome than group B $(p<0.05)$.

Conclusions: The selective nerve root block showed superior results up to 6 weeks post-procedure. Considering that the selective nerve root block is effective for treating radiculopathy, the primary cause of buttock pain can be thought to be radiculopathy rather than degenerative changes of the facet joint.

Keywords: Buttocks, Pain, Nerve block, Spinal stenosis

Patients with spinal stenosis, one of the most common degenerative diseases of the spine, present with lumbar pain, buttock pain, and pain radiating to the lower legs. Buttock pain can be caused by a stimulus to the affected lumbar spinal nerve, resulting in radiating pain in the respective

Received October 29, 2018; Accepted November 26, 2018

Correspondence to: Chang Su Kim, MD

Department of Orthopaedic Surgery, Kosin University Gospel Hospital,

262 Gamcheon-ro, Seo-gu, Busan 49267, Korea

Tel: +82-51-990-6467, Fax: +82-51-243-0181

E-mail: mewha98@naver.com dermatome, or by referred pain. ${ }^{1)}$

Goebert et al. ${ }^{2)}$ reported that inflammation around the nerve root causes lumbar pain and lower leg radiating pain; thus, steroid and procaine injections can lead to symptomatic improvement. After Cooper et al. ${ }^{3)}$ introduced a selective nerve root block for degenerative lumbar scoliosis, studies have demonstrated improvements in short-term and long-term outcomes. ${ }^{4,5}$

Eubanks et al. $\left.{ }^{6}\right)$ reported that a facet joint block induced clinically significant improvements in buttock pain, morning stiffness, and lumbar pain after the procedure. Mooney and Robertson ${ }^{7)}$ reported that a facet joint block 
Shim et al. Buttock Pain in Spinal Stenosis Patients

Clinics in Orthopedic Surgery • Vol. 11, No. 1, $2019 \bullet$ www.ecios.org

led to improvements in $62 \%$ of patients during an early follow-up and $20 \%$ of patients during a long-term followup.

Many studies, including the one by Donovan et al., ${ }^{8)}$ reported that facet joint lesions are associated with axial pain of the thoracolumbar area and referred pain to the limbs. This referred pain can have varying effects on patients with identical locations of lesions and even varying effects on one individual at different times of the day, which can cause diagnostic confusion. Generally, thorough history taking and recognition of symptoms are required for the differential diagnosis. However, Laplante et al. ${ }^{9}$ stated that discogenic, facet joint, and sacroiliac joint pain, which mediate lumbar pain, are related to overlapping pain, i.e., radiating pain to the lower legs. Multivariate analysis could not differentiate the etiology based on the presence of radiating pain to the lower legs. Physical examination and imaging studies can be useful, but it remains difficult to confirm the diagnosis because the characteristics of pain cannot be easily identified clinically.

In practice, buttock pain is very common, and there is no set guideline for its diagnosis and treatment. In the past, studies have investigated lower leg radiating pain or lumbar pain, but no study has investigated the etiology of buttock pain or its treatment.

This article attempted to make a retrograde inference of the etiology of pain by comparing the treatment outcome of patients with buttock pain receiving different types of nerve blocks. If buttock pain is improved after selective nerve root blocks, then the pain is likely to be caused by radiculopathy. If the pain is relieved by facet joint blocks, then the pain is likely to be caused by degenerated facet joints.

\section{METHODS}

\section{Patients}

This prospective study targeted 191 patients with buttock pain who visited Kosin University Gospel Hospital from June 2017 to September 2017, with at least 3 weeks of Quebec Task Force category I or II pain, and who could be followed up for more than 3 months. Imaging tests using X-ray and magnetic resonance imaging (MRI) were conducted in all patients of this article. Patients in this research only had buttock pain and no radiating pain. Either a selective nerve root block (group A) or a facet joint block (group B) was conducted on patients with lumbar central canal stenosis grade 2 or above and patients with lumbar foraminal stenosis grade 2 or above..$^{10,11)}$

Exclusion criteria were as follows: patients with a single herniated nucleus pulposus with concurrent symptoms with dermatome and myotome involvement confirmed by MRI, those with sagittal spinal imbalance of more than $10 \mathrm{~cm}$ in front of the posterosuperior corner of the S1 vertebral body from the C7 plumb line, those with an acute fracture, those with paralysis, and those with a prominent hip joint lesion on physical examination and imaging studies. In total, 146 patients met the study inclusion criteria; 76 patients who were treated with the selective nerve root block were assigned to group $\mathrm{A}$, and 70 patients who were treated with the facet joint block were assigned to group B.

\section{Study Interventions}

The blocks were performed by a single skilled physician (CSK). The location of the facet joint block was selected based on local tender points and the tender points identified by the patient. When local tenderness was prominent, the physician determined the injection point based on the severity of tenderness and the laterality of the pain. After the joint was selected, patients were placed in the prone position with a pillow underneath the abdomen, thus inducing kyphosis of the lumbar spine. With a posterior approach, the articular processes were widened, sterilized according to routine procedures, and evaluated under fluorescent light. Local anesthetic with $1 \%$ lidocaine was performed. Then, injection was performed using a 22-25$G$ needle at the inferior articular process of the facet joint. A tenderness provocation test was performed using 1-3 $\mathrm{mL}$ of a non-neurotoxic contrast (water-soluble contrast media; Telebrix, Schering, Berlin-Wedding, Germany). Anterior-posterior and lateral plain radiographs were obtained to identify the shape of the joint. Then, $0.5 \mathrm{~mL}$ of lidocaine and $1 \mathrm{~mL}$ of dexamethasone were injected into the joint. When multiple joints were treated, the amount of dexamethasone was the same, and $1 \mathrm{~mL}$ of lidocaine was mixed per joint. No more than three vertebrae were treated with injection at the same time.

Nerve root blocks were performed at the prospective spinal root lesion assisted by the use of plain radiographs, computed tomography, or MRI. In principle, one spinal nerve root was treated during each session, but when the symptoms were present bilaterally, two treatments were provided during the same session. The vertebral nerve root block was performed using the posterior approach. The patient was placed in a prone position on the radiation table and treated with local anesthesia. Using fluoroscopy, a $22-\mathrm{G}$ spinal needle was inserted from a point located $4 \mathrm{~cm}$ lateral to the superior lumbar spinous process to a point located on the lateral side of the vertebral body and the 
inferior transverse process at $30^{\circ}-45^{\circ}$ angle from the sagittal plain caudally so that the needle passed through the entrance of the intervertebral foramen. However, in case of S1 nerve root block, because of the anatomical structure of the sacrum, the needle was inserted slowly into the first sacral foramina at an approximately $10^{\circ}-15^{\circ}$ oblique angle in the caudal direction in order to reach the first sacral nerve. When the end of the needle stimulated the nerve root, the patient would feel a sharp radiating pain or numbness. At this point, $0.5 \mathrm{~mL}$ of water-soluble contrast media (Telebrix) was injected to identify the traction of the nerve root, and a mixture of $0.5 \mathrm{~mL}$ of dexamethasone and $0.5 \mathrm{~mL}$ of lidocaine was injected. After the selective nerve root block, bed rest for 3 hours was prescribed until the resolution of numbness, desensitization, and asthenia.

In order to prevent infection during the treatment, all procedures were performed as aseptically as possible. Patients who were older in age or with lowered immune function were treated with additional caution. Treatment for iatrogenic infection was planned in advance.

\section{Outcome Measures and Statistical Analysis}

Evaluation was performed before the procedure and on post-procedure day 1 , week 2 , week 6 , and week 12 using the visual analog scale (VAS) and the Korean version of the Oswestry Disability Index (K-ODI) 2.0. The evaluation was performed according to the following scale: poor, VAS score improved by less than 1 point; fair, VAS score improved by 1-2 points; good, VAS score improved by 3-4 points; and excellent, VAS score improved by greater than 5 points. Then, the results were compared according to the method of injection.

Statistical analysis was performed using SPSS ver. 12.0 (SPSS Inc., Chicago, IL, USA), and the control values were compared using the $t$-test. All values are reported as a mean and standard deviation. Because of the limited number of patients, the results did not follow a chi-square distribution; thus, $2 \times 2$ cross-table analysis was performed using Fisher exact test to analyze the response to injection.

\section{RESULTS}

In group A, 28 patients were male and 48 were female. Patients' mean age was 65 years (range, 34 to 87 years), and the mean follow-up period was 5.3 months. In group B, 27 patients were male and 43 were female. Patients' mean age was 65 years (range, 35 to 85 years), and the mean followup period was 5.5 months. There was no significant difference between groups in terms of sex, age, or underlying diseases (Table 1).

One day after the procedure, an excellent response was found in 7\% of group A and in 6\% of group B, and a good response was found in $41 \%$ of group $A$ and in $13 \%$ of group B. Two weeks after the procedure, an excellent

\begin{tabular}{lccc}
\hline \multicolumn{1}{|c|}{ Table 1. Demographic Data } \\
Variable & $\begin{array}{c}\text { Group A } \\
(\mathrm{n}=76)\end{array}$ & $\begin{array}{c}\text { Group B } \\
(\mathrm{n}=70)\end{array}$ & $p$-value \\
\hline Age (yr) & 65.71 & 65.03 & $>0.05$ \\
Sex (male:female) & $28: 48$ & $27: 43$ & $>0.05$ \\
DM & $18(20)$ & $18(26)$ & $>0.05$ \\
\hline HTN & $33(43)$ & $28(40)$ & $>0.05$ \\
\hline
\end{tabular}

Values are presented as number (\%).

Group A: selective nerve root block, Group B: facet joint block, DM: diabetes mellitus, HTN: hypertension.
A

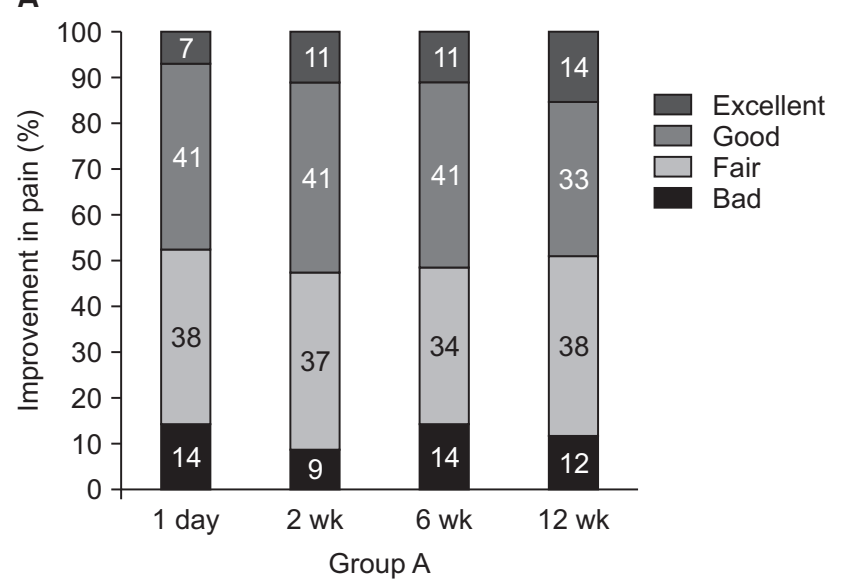

B

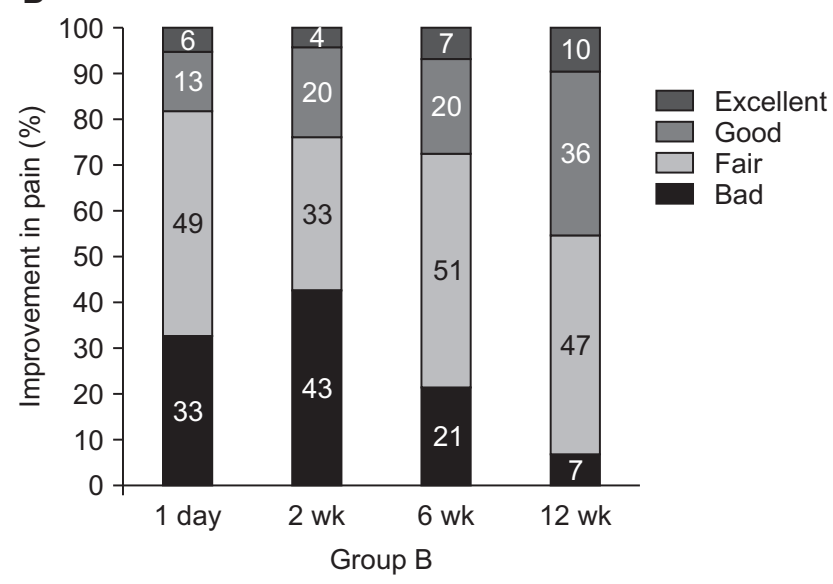

Fig. 1. Comparison of the improvement in pain. Group A: selective nerve root block, Group B: facet joint block. 
Shim et al. Buttock Pain in Spinal Stenosis Patients

Clinics in Orthopedic Surgery • Vol. 11, No. 1, 2019•www.ecios.org

\section{Table 2. VAS Scores}

\begin{tabular}{lccc}
\multicolumn{1}{c}{ VAS score } & $\begin{array}{c}\text { Group A } \\
(\mathrm{n}=76)\end{array}$ & $\begin{array}{c}\text { Group B } \\
(\mathrm{n}=70)\end{array}$ & $p$-value \\
\hline Preoperative & $5.01 \pm 2.06$ & $5.24 \pm 2.03$ & 0.926 \\
\hline Postoperative 1 day & $2.74 \pm 1.24$ & $3.94 \pm 1.81$ & $<0.05$ \\
\hline Postoperative 2 wk & $2.51 \pm 1.25$ & $3.99 \pm 1.73$ & $<0.05$ \\
\hline Postoperative 6 wk & $2.38 \pm 1.26$ & $3.24 \pm 1.75$ & $<0.05$ \\
\hline Postoperative 12 wk & $2.39 \pm 1.30$ & $2.59 \pm 1.44$ & 0.27 \\
\hline
\end{tabular}

Values are presented as mean \pm standard deviation.

VAS: visual analog scale, Group A: selective nerve root block, Group B: facet joint block.

response was found in $11 \%$ of group A and in $4 \%$ of group $\mathrm{B}$, and a good response was found in $41 \%$ of group $\mathrm{A}$ and in $20 \%$ of group B. Six weeks after the procedure, an excellent response was found in $11 \%$ of group $\mathrm{A}$ and in $7 \%$ of group B, and a good response was found in $41 \%$ of group A and in $20 \%$ of group B. At the final follow-up, 12 weeks after the procedure, $47 \%$ showed a good response in group A, and $46 \%$ showed a good response in group B (Fig. 1).

In group A, VAS improved compared to the preprocedure value of 5.01 to 2.74 on day $1,2.51$ at week $2,2.38$ at week 6, and 2.39 at week 12. In group B, the VAS score improved compared to the pre-procedure value of 5.24 to 3.94 on day $1,3.99$ at week $2,3.24$ at week 6 , and 2.59 at week 12. On day 1 and at weeks 2 and 6 , group A showed a significantly better outcome than group B $(p<0.05)$ (Table 2).

In group A, K-ODI improved compared to the preprocedure value of $34.03 \pm 5.42$ to $15.68 \pm 3.00$ on day 1 , $15.21 \pm 2.28$ at week $2,16.11 \pm 2.69$ at week 6 , and $17.08 \pm$ 3.31 at week 12. In group B, K-ODI showed a trend toward improvement compared to the pre-procedure value of $34.65 \pm 5.28$ to $17.66 \pm 3.54$ on day $1,17.94 \pm 3.21$ at week $2,18.49 \pm 3.29$ at week 6 , and $18.43 \pm 3.26$ at week 12 . On day 1 and at weeks 2 and 6, group A showed significantly better outcome in terms of symptom relief than group B ( $p$ $<0.05$ ) (Table 3).

\section{DISCUSSION}

Macnab and MacCulloch ${ }^{12)}$ categorized degenerative diseases of the spine according to the etiology of pain as psychogenic, viscerogenic, vascular, neurogenic, and spondylogenic. Degenerative diseases of the spine such as spinal stenosis, facet joint syndrome, lumbosacral arthritis, and hip joint-related disorders including greater

\begin{tabular}{|cccc}
\hline Table 3. K-ODI 2.0 & & & \\
\hline \multicolumn{1}{c}{ K-ODI } & $\begin{array}{c}\text { Group A } \\
(\mathrm{n}=76)\end{array}$ & $\begin{array}{c}\text { Group B } \\
(\mathrm{n}=70)\end{array}$ & $p$-value \\
\hline Preoperative & $34.03 \pm 5.42$ & $34.65 \pm 5.28$ & 0.833 \\
\hline Postoperative 1 day & $15.68 \pm 3.00$ & $17.66 \pm 3.54$ & $<0.05$ \\
\hline Postoperative 2 wk & $15.21 \pm 2.28$ & $17.94 \pm 3.21$ & $<0.05$ \\
\hline Postoperative 6 wk & $16.11 \pm 2.69$ & $18.49 \pm 3.29$ & $<0.05$ \\
\hline Postoperative 12 wk & $17.08 \pm 3.31$ & $18.43 \pm 3.26$ & 0.668 \\
\hline
\end{tabular}

Values are presented as mean \pm standard deviation.

K-ODI: Korean version of the Oswestry Disability Index, Group A: selective nerve root block, Group B: facet joint block.

trochanter syndrome, iliotibial band syndrome, hip osteoarthritis, and avascular necrosis can all cause buttock pain. Therefore, it is crucial to identify the underlying etiology of buttock pain. Patients with spinal stenosis more often present with buttock pain as a chief complaint rather than radiating pain to the legs. Additionally, patients with facet joint syndrome can have lumbar pain and buttock pain. However, it is unclear if buttock pain in degenerative spine disease is caused by spinal stenosis or facet joint syndrome. Mooney and Robertson ${ }^{7)}$ argued that the facet joint plays an important role in causing lumbar pain. Robinson et al. ${ }^{13)}$ questioned the effects of anti-inflammatory injection to the facet joint. Krempen et al. ${ }^{1)}$ reported that a nerve root block should be used for patients whose radiological evaluations cannot fully identify the cause of pain or differentiate the characteristic of pain as referred or radiating.

The facet joint is innervated by the medial branch of the dorsal ramus of the nerve originating from the superior segment and the corresponding segment; the nerve to the facet joint is a nociceptive receptor. Marks ${ }^{14)}$ reported that based on clinical facet joint injection, buttock pain usually originates at the L4-5 level. On the other hand, inguinal pain usually originates at the L $2-5$ level. The nerve that innervates the facet joint produces referred distal pain more prominently than joint pain itself. Mehta and Sluijter ${ }^{15)}$ argued that even if there is no evident degenerative change in the facet joint detected by radiography, a patient can have lumbar pain, and lumbar pain is generated from the capsule rather than the frame of the facet joint.

Radiating pain is mediated through the anterior primary ramus. Bernard and Kirkaldy-Willis ${ }^{16)}$ reported that radiating pain occurs at the waist, buttock, calf along the sciatic nerve, or the anterior thigh along the femoral nerve. Allegri et al. ${ }^{17)}$ also reported that inflammation plays a more important role than direct compression of the nerve 
root in pathological studies of radiating pain.

The present study focused on identifying the cause of buttock pain as either radiating pain or facet joint degeneration by using a retrograde deduction method. In the evaluation, patients treated with the selective nerve root block showed a significantly superior outcome compared to patients treated with the facet joint block on postprocedure day 1 , week 2 , week 6 , and week 12 . Based on the finding that the selective nerve root block improved pain, the cause of buttock pain is likely to be radiculopathy rather than degenerative changes of the facet joint.

Krames $^{18)}$ stated that stimulation to the dorsal root ganglion (DRG) plays an important role in the generation of neuropathic pain and that the DRG is a clinical target for pain control. Buttock pain could be caused by stimulation of the DRG, and a nerve root block can affect the DRG and thus alleviate pain.

There are limitations to this study. Patients were also treated with medication after the procedure, and there were factors that interfered with the single injection treatment. The characteristic of the pain was not well described or considered in the analysis. It is possible that the buttock pain is due to radiculopathy or referred pain. On the basis of this study, we can conclude that radiculopathy is a more frequent cause of buttock pain than referred pain, although a neurophysiology study must be done to demonstrate a direct connection. Additionally, our study population was small, and further large-scale trials are warranted.

In conclusion, for buttock pain, the selective nerve root block showed a significantly superior result up to 6 weeks after the procedure compared to the facet joint block. Considering that the selective nerve root block is effective for radiculopathy, the primary cause of buttock pain can be considered to be radiculopathy rather than degenerative changes of the facet joint.

\section{CONFLICT OF INTEREST}

No potential conflict of interest relevant to this article was reported.

\section{ACKNOWLEDGEMENTS}

This research was supported by a grant from Wonkwang University, Iksan, Korea, in 2018.

\section{REFERENCES}

1. Krempen JF, Smith BS, DeFreest LJ. Selective nerve root infiltration for the evaluation of sciatica. Orthop Clin North Am. 1975;6(1):311-5.

2. Goebert HW Jr, Jallo SJ, Gardner WJ, Wasmuth CE. Painful radiculopathy treated with epidural injections of procaine and hydrocortisone acetate: results in 113 patients. Anesth Analg. 1961;40:130-4.

3. Cooper G, Lutz GE, Boachie-Adjei O, Lin J. Effectiveness of transforaminal epidural steroid injections in patients with degenerative lumbar scoliotic stenosis and radiculopathy. Pain Physician. 2004;7(3):311-7.

4. Hong YG, Sa SJ, Kim JD. Selective spinal nerve root block for the treatment of sciatica. J Korean Orthop Assoc. 1997;32(4):1056-62.

5. Narozny M, Zanetti M, Boos N. Therapeutic efficacy of selective nerve root blocks in the treatment of lumbar radicular leg pain. Swiss Med Wkly. 2001;131(5-6):75-80.

6. Eubanks JD, Lee MJ, Cassinelli E, Ahn NU. Prevalence of lumbar facet arthrosis and its relationship to age, sex, and race: an anatomic study of cadaveric specimens. Spine (Phila Pa 1976). 2007;32(19):2058-62.

7. Mooney V, Robertson J. The facet syndrome. Clin Orthop
Relat Res. 1976;(115):149-56.

8. Donovan WH, Dwyer AP, White BW, Batalin NJ, Skerritt PW, Bedbrook GM. A multidisciplinary approach to chronic low-back pain in Western Australia. Spine (Phila Pa 1976). 1981;6(6):591-7.

9. Laplante BL, Ketchum JM, Saullo TR, DePalma MJ. Multivariable analysis of the relationship between pain referral patterns and the source of chronic low back pain. Pain Physician. 2012;15(2):171-8.

10. Lee GY, Lee JW, Choi HS, Oh KJ, Kang HS. A new grading system of lumbar central canal stenosis on MRI: an easy and reliable method. Skeletal Radiol. 2011;40(8):1033-9.

11. Lee S, Lee JW, Yeom JS, et al. A practical MRI grading system for lumbar foraminal stenosis. AJR Am J Roentgenol. 2010;194(4):1095-8.

12. Macnab I, MacCulloch J. Backache. 2nd ed. Baltimore: Williams and Wilkins; 1990. 22-5.

13. Robinson JP, Brown PB, Fisk JD. Pathophysiology of lumbar radiculopathies and the pharmacology of epidural corticosteroids and local anesthetics. Phys Med Rehabil Clin N Am. 1995;6(4):671-90.

14. Marks R. Distribution of pain provoked from lumbar facet 
Shim et al. Buttock Pain in Spinal Stenosis Patients

Clinics in Orthopedic Surgery • Vol. 11, No. 1, 2019•www.ecios.org

joints and related structures during diagnostic spinal infiltration. Pain. 1989;39(1):37-40.

15. Mehta M, Sluijter ME. The treatment of chronic back pain: a preliminary survey of the effect of radiofrequency denervation of the posterior vertebral joints. Anaesthesia. 1979;34(8):768-75.

16. Bernard TN Jr, Kirkaldy-Willis WH. Recognizing specific characteristics of nonspecific low back pain. Clin Orthop
Relat Res. 1987;(217):266-80.

17. Allegri M, Montella S, Salici F, et al. Mechanisms of low back pain: a guide for diagnosis and therapy. F1000Res. 2016;5:1530.

18. Krames ES. The dorsal root ganglion in chronic pain and as a target for neuromodulation: a review. Neuromodulation. 2015;18(1):24-32. 